\title{
Numerical Solutions of the Regularized Long-Wave (RLW) Equation Using New Modification of Laplace-Decomposition Method
}

\author{
Nawal A. Al-Zaid, Huda O. Bakodah, Fathiah A. Hendi \\ Department of Mathematics, Science Faculty for Girls, King Abdulaziz University, Jeddah, KSA \\ Email: nalzaid@kau.edu.sa,hbakodah@kau.edu.sa, falhendi@kau.edu.sa
}

Received July 28, 2012; revised September 30, 2012; accepted October 8, 2012

\begin{abstract}
In this paper the new modification of Laplace Adomian decomposition method (ADM) to obtain numerical solution of the regularized long-wave (RLW) equation is presented. The performance of the method is illustrated by solving two test examples of the problem. To see the accuracy of the method, $L_{2}$ and $L_{\infty}$ error norms are calculated.
\end{abstract}

Keywords: Adomian Decomposition Method; Regularized Long-Wave (RLW); Laplace Transform

\section{Introduction}

The regularized long wave (RLW) equation which can be shown in the form

$$
u_{t}+u_{x}+\epsilon u u_{x}-\mu u_{x x t}=0
$$

where $\epsilon, \mu$ are positive parameters, is an important nonlinear wave equation. This equation plays a major role in the study of nonlinear dispersive waves. The RLW equation particularly describes the behavior of the undular bore [1-3], it has also been derived from the study of water waves and ion acoustic plasma waves.

The RLW equation has been solved analytically only for restricted set of boundary and initial conditions. Therefore, the numerical solution of this equation has been the subject of many papers [4-7]. Recently a great deal of interest has been focused on application of Adomian decomposition method (ADM) to solve a wide variety of nonlinear problems $[8,9]$. In this paper, we will apply the new modification of Laplace ADM to the RLW Equation (1). The soliton solution of RLW equation has the form

$$
u(x, t)=3 c \operatorname{sech}^{2}\left(p\left(x-v t-x_{0}\right)\right)
$$

where $p$ is an arbitrary constant and $p=\frac{1}{2}\left(\frac{c}{\mu(1+c)}\right)^{\frac{1}{2}}$, $v=c+1, \varepsilon=1$, and $c>0$ is a constant [10]. In this work, a new modification of Laplace ADM is used to solve the RLW equation with the initial condition

$$
u(x, 0)=f(x)
$$

where $f(x)$ is a localized disturbance inside the con- sidered interval.

\section{Description of Method}

We begin by consider Equation (1) in an operator form

$$
L_{t} u+u_{x}-\mu R(u)+\epsilon N(u)=0
$$

where $\left(L_{t}=\frac{\partial}{\partial t}\right)$ is a linear operator and $R$ its remainder of the linear operator. The nonlinear term is represented by $N(u)$. Thus we get

$$
L_{t} u=-u_{x}+\mu R(u)-\epsilon N(u)
$$
low,

We represent solution as an infinite series given be-

$$
u(x, t)=\sum_{n=0}^{\infty} u_{n}(x, t)
$$

The nonlinear term $\mathrm{Nu}$ can be decomposed into infinite series of polynomial given by:

$$
N u=u u_{x}=\sum_{n=0}^{\infty} A_{n}
$$

where $A_{n}$ are Adomian polynomials [11] of $u_{0}, u_{1}, \cdots, u_{n}$ and it can be calculated by formula given below:

$$
\begin{gathered}
A_{0}=u_{0 x} u_{0} \\
A_{1}=u_{0 x} u_{1}+u_{1 x} u_{0} \\
A_{2}=u_{0 x} u_{2}+u_{1 x} u_{1}+u_{2 x} u_{0}
\end{gathered}
$$

and so on. The rest of the polynomials can be constructed 
in a similar manner.

By applying the Laplace transform to both sides of Equation (5), we obtain

$$
\mathcal{L}\left\{L_{t}(u)\right\}=-\mathcal{L}\left\{u_{x}\right\}+\mu \mathcal{L}\{R u\}-\epsilon \mathcal{L}\{N u\}
$$

Thus

$$
\mathcal{L}\{u(x, t)\}=\frac{1}{s} u(x, 0)-\frac{1}{s}\left(\mathcal{L}\left\{u_{x}\right\}+\mu \mathcal{L}\{R u\}-\epsilon \mathcal{L}\{N u\}\right)
$$

In the new modification of ADM [12], Wazwaz replaced the initial condition $u(x, 0)$ by a series of infinite components i.e.,

$$
u(x, 0)=\sum_{n=0}^{\infty} u_{0, n}(x)
$$

and the new recursive relationship can be expressed in the form

$$
\begin{aligned}
U_{0}(x, s)= & \frac{1}{s} u_{0,0}(x, 0), \\
U_{n+1}(x, s)= & \frac{1}{s} u_{0, n+1}(x, 0) \\
& -\frac{1}{s}\left(\mathcal{L}\left\{u_{x}\right\}+\mu \mathcal{L}\{R u\}-\epsilon \mathcal{L}\{N u\}\right), n \geq 0
\end{aligned}
$$

where

$$
\mathcal{L}\{u(x, t)\}=U(x, s)=\sum_{n=0}^{\infty} U_{n}(x, s)
$$

Now, by applying inverse Laplace transformation we get:

$$
\begin{aligned}
& u_{0}(x, t)=\mathcal{L}^{-1}\left\{U_{0}(x, s)\right\} \\
& u_{n+1}(x, t)=\mathcal{L}^{-1}\left\{U_{n+1}(x, s)\right\}, n \geq 0
\end{aligned}
$$

Using (13) the series solution follows immediately.

\section{Numerical Examples and Results}

In this section, the new modification of Laplace ADM will be demonstrated on illustrative examples and we compare the approximate solution obtained for our RLW equation with known exact solutions. We define $u_{m}$ to be m-term approximate solution, i.e.

$$
u_{m}=\sum_{i=0}^{m} u_{i}(x, t)
$$

$u_{e}$ the exact solution and $e_{m}$ the absolute error between the exact solution and the approximate solution

$$
e_{m}=\left|u_{e}-u_{m}\right|
$$

In order to show how good the numerical solutions are in comparison with the exact ones, we will use the $L_{2}$ and $L_{\infty}$ error norms defined by

$$
\begin{gathered}
L_{2}=u_{e}-u_{m 2}=\left[\Delta x \sum_{i=1}^{m}\left|u_{e, i}-u_{m, i}\right|^{2}\right]^{\frac{1}{2}} \\
L_{\infty}=\left\|u_{e}-u_{m}\right\|_{\infty}=\max _{i}\left|u_{e, i}-u_{m, i}\right|
\end{gathered}
$$

\section{Example (1)}

We consider Equation (1) with the initial condition

$$
u(x, 0)=3 \operatorname{csech}^{2}\left(p\left(x-x_{0}\right)\right)
$$

The exact solution of this problem is given by Equation (2). This solution corresponds to the motion of a single solitary wave with amplitude $3 c$ and width $p$, initially centered at $x_{0}$, where $v=1+c$ is the wave velocity. We use the new modification of Laplace ADM to solve this equation, all computations are done for the parameters $\varepsilon=1, \mu=1$ and $x_{0}=0$.

We consider $c=0.1$, as in [13], so the initial condition $u(x, 0)$ can expressed as a series of infinite components i.e.

$$
\begin{aligned}
u(x, 0)= & 0.3-0.00681818 x^{2}+0.000103306 x^{4} \\
& -1.33045 x^{6}+1.5754 x^{8}-1.77355 x^{10}+O[x]^{12}
\end{aligned}
$$

Using recursive relation (11) yield the components

$$
\begin{aligned}
u_{0}(x, t)= & \mathcal{L}^{-1}\left\{U_{0}(x, s)\right\}=\mathcal{L}^{-1}\left\{\frac{0.3}{s}\right\}=0.3 \\
u_{1}(x, t)= & \mathcal{L}^{-1}\left\{U_{1}(x, s)\right\}=\mathcal{L}^{-1}\left\{\frac{-0.00681818}{s} x^{2}\right\} \\
= & -0.00681818 x^{2} \\
u_{2}(x, t)= & \mathcal{L}^{-1}\left\{U_{2}(x, s)\right\} \\
= & 0.0177273 t x+0.000103306 x^{4} \\
u_{3}(x, t)= & \mathcal{L}^{-1}\left\{U_{3}(x, s)\right\} \\
= & -0.0115227 t^{2}-0.000630165 t x^{3} \\
& -1.33045(10)^{-6} x^{6}
\end{aligned}
$$

And so on, in this manner the rest of components of the decomposition series were obtained. The results are given in Table 1. The error norms for $(c=0.1)$ are recorded in Table 2 for different value of $m$.

Also in Figure 1 we show the exact solution and numerical solution with new modification of Laplace ADM for $t=0.1$ and $t=0.5$. Figure 2 shows the exact solution and numerical solution with new modification for $t=0.5$ at the interval $5 \leq x \leq 5$.

\section{Example (2)}

In the second test problem [14], a smaller solitary wave of amplitude 0.109 ( $c=0.05)$, has been modeled.

The initial condition $u(x, 0)$ can expressed as a series of infinite components i.e. 
Table 1. Absolute errors for Example (1) with $c=0.1$ and $m=10$.

\begin{tabular}{cccccc}
\hline$x / t$ & 0.01 & 0.02 & 0.03 & 0.04 & 0.05 \\
\hline 0.1 & $4.60152 \times 10^{-7}$ & $4.29123 \times 10^{-7}$ & $9.14459 \times 10^{-8}$ & $1.09975 \times 10^{-6}$ & $2.59382 \times 10^{-6}$ \\
0.2 & $1.15346 \times 10^{-6}$ & $1.8112 \times 10^{-6}$ & $1.97626 \times 10^{-6}$ & $1.65183 \times 10^{-6}$ & $8.41272 \times 10^{-7}$ \\
0.3 & $1.82435 \times 10^{-6}$ & $3.1446 \times 10^{-6}$ & $3.96518 \times 10^{-6}$ & $4.29071 \times 10^{-6}$ & $4.12595 \times 10^{-6}$ \\
0.4 & $2.4624 \times 10^{-6}$ & $4.40862 \times 10^{-6}$ & $5.84456 \times 10^{-6}$ & $6.77623 \times 10^{-6}$ & $7.20981 \times 10^{-6}$ \\
0.5 & $3.05691 \times 10^{-6}$ & $5.58214 \times 10^{-6}$ & $7.58301 \times 10^{-6}$ & $9.06702 \times 10^{-6}$ & $1.00418 \times 10^{-5}$ \\
\hline
\end{tabular}

Table 2. $L_{2}$ and $L_{\infty}$ errors for Example (1) with $m=4,6$ and 10.

\begin{tabular}{|c|c|c|c|c|c|c|}
\hline \multirow{2}{*}{$\frac{n}{x}$} & \multicolumn{2}{|c|}{4} & \multicolumn{2}{|c|}{6} & \multicolumn{2}{|c|}{10} \\
\hline & $L_{2}$ & $L_{\infty}$ & $L_{2}$ & $L_{\infty}$ & $L_{2}$ & $L_{\infty}$ \\
\hline 0.1 & $7.08453 \times 10^{-7}$ & $5.4329 \times 10^{-6}$ & $2.30062 \times 10^{-7}$ & $2.44677 \times 10^{-7}$ & $1.88979 \times 10^{-6}$ & $2.59382 \times 10^{-6}$ \\
\hline 0.2 & $1.71987 \times 10^{-6}$ & $1.8958 \times 10^{-5}$ & $9.54522 \times 10^{-7}$ & $5.43345 \times 10^{-6}$ & $9.42236 \times 10^{-7}$ & $8.41272 \times 10^{-7}$ \\
\hline 0.3 & $2.7237 \times 10^{-6}$ & $3.2340 \times 10^{-5}$ & $1.70526 \times 10^{-6}$ & $1.05194 \times 10^{-5}$ & $9.18072 \times 10^{-7}$ & $4.12595 \times 10^{-6}$ \\
\hline 0.4 & $3.71444 \times 10^{-6}$ & $4.5525 \times 10^{-5}$ & $2.43975 \times 10^{-6}$ & $1.54669 \times 10^{-5}$ & $1.73297 \times 10^{-6}$ & $7.20981 \times 10^{-6}$ \\
\hline 0.5 & $4.6877 \times 10^{-6}$ & $5.8456 \times 10^{-5}$ & $3.15018 \times 10^{-6}$ & $2.02399 \times 10^{-5}$ & $2.61274 \times 10^{-6}$ & $1.00418 \times 10^{-5}$ \\
\hline
\end{tabular}

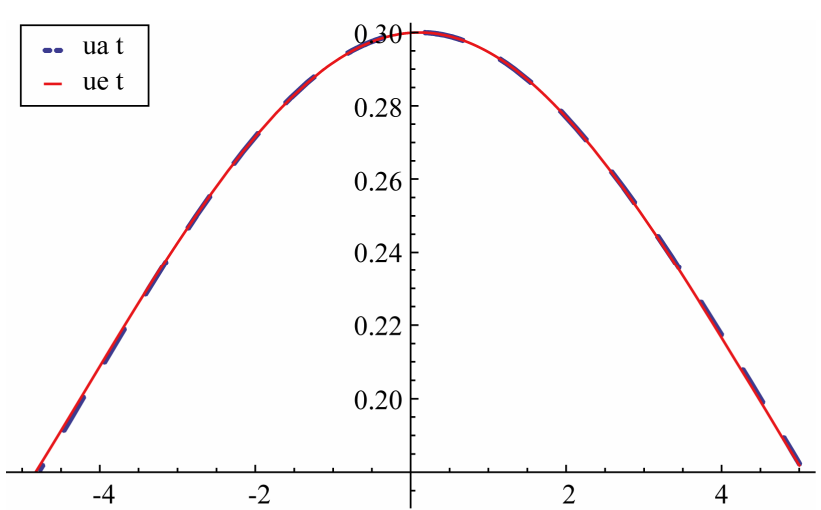

(a)

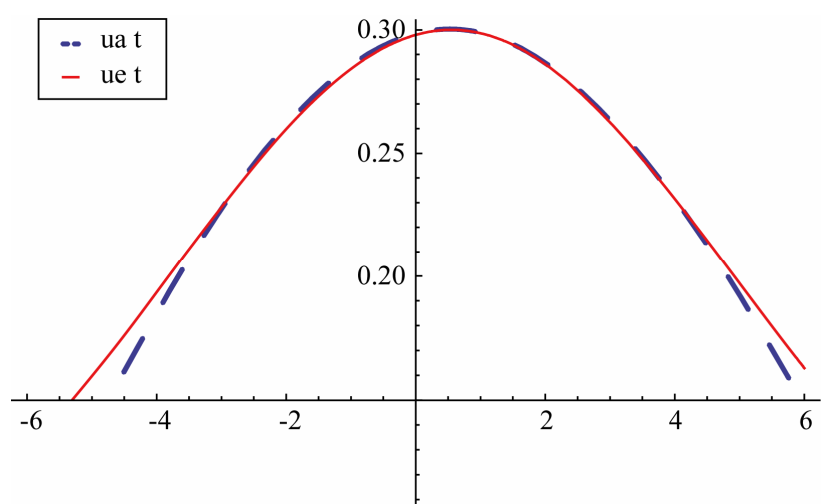

(b)

Figure 1. The exact solution and numerical solution with new modification of Laplace ADM for Example (1), for $t=0.1$ and (b) for $t=0.5$.

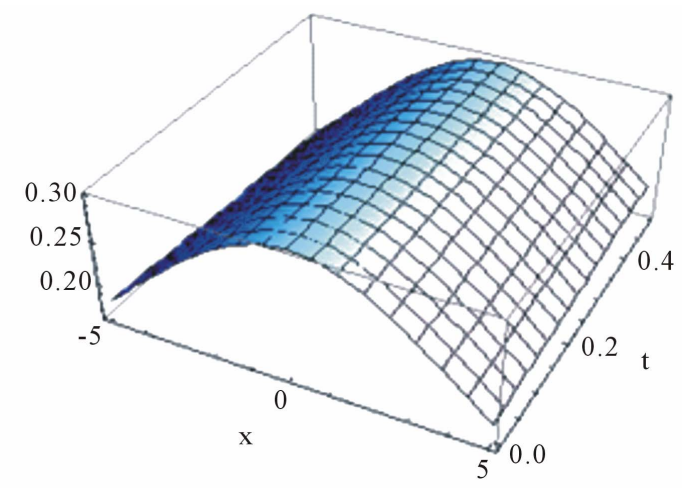

(a)

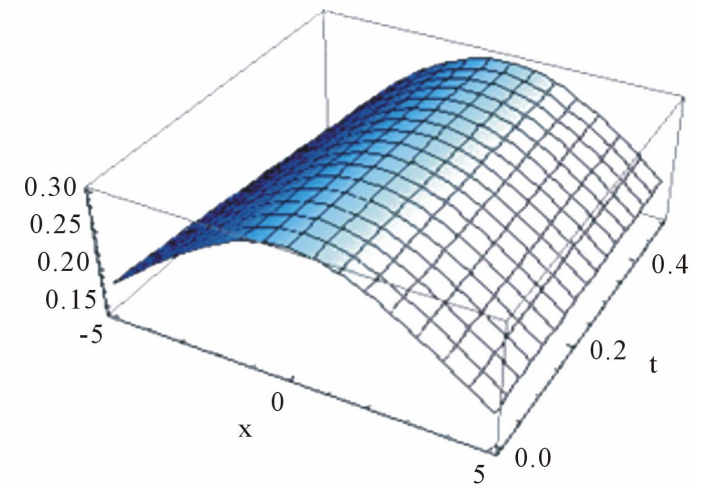

(b)

Figure 2. (a) The exact solution for $t=0.5$ and $-5 \leq x \leq 5$; (b) Numerical solution with new modification for $t=0.5$ and $-5 \leq x$ $\leq 5$. 


$$
\begin{aligned}
u(x, 0)= & 0.15-0.00178571 x^{2} \\
& +0.0000141723 x^{4}-9.5607 \times(10)^{-8} x^{6} \\
& +5.93001 \times(10)^{-10} x^{8}+O[x]^{12}
\end{aligned}
$$

Using recursive relation (11) yield the components

$$
\begin{aligned}
u_{0}(x, t) & =\mathcal{L}^{-1}\left\{U_{0}(x, s)\right\} \\
& =\mathcal{L}^{-1}\left\{\frac{0.15}{s}\right\}=0.15 \\
u_{1}(x, t) & =\mathcal{L}^{-1}\left\{U_{1}(x, s)\right\}=\mathcal{L}^{-1}\left\{\frac{-0.00178571}{s} x^{2}\right\} \\
& =-0.00178571 x^{2} \\
u_{2}(x, t) & =\mathcal{L}^{-1}\left\{U_{2}(x, s)\right\} \\
& =0.00410714 t x+0.0000141723 x^{4}
\end{aligned}
$$

$$
\begin{aligned}
u_{3}(x, t)= & \mathcal{L}^{-1}\left\{U_{3}(x, s)\right\} \\
= & -0.00236161 t^{2}-0.0000715703 t x^{3} \\
& -9.5607 \times(10)^{-8} x^{6}
\end{aligned}
$$

And so on, in this manner the rest of components of the decomposition series were obtained.

The results are given in Table $\mathbf{3}$ and the error norms for $(c=0.05)$ are recorded in Table 4 for different value of $m$. Also in Figure 3 we show the exact solution and numerical solution with new modification of Laplace ADM for $t=0.1$ and $t=0.5$. Figure 4 show the exact solution and numerical solution with new modification for $t=0.5$ at the interval $10 \leq x \leq 10$.

\section{Conclusion}

In this paper, we use the new modification of Laplace

Table 3. Absolute errors for Example (2) with $c=0.05, k=0.109$ and $m=10$.

\begin{tabular}{cccccc}
\hline$x / t$ & 0.01 & 0.02 & 0.03 & 0.04 & 0.05 \\
\hline 0.1 & $5.68488 \times 10^{-9}$ & $2.48779 \times 10^{-9}$ & $9.54769 \times 10^{-9}$ & $3.03715 \times 10^{-8}$ & $5.99272 \times 10^{-8}$ \\
0.2 & $1.56382 \times 10^{-8}$ & $2.23134 \times 10^{-8}$ & $2.01032 \times 10^{-8}$ & $9.09157 \times 10^{-9}$ & $1.06314 \times 10^{-8}$ \\
0.3 & $2.52853 \times 10^{-8}$ & $4.14523 \times 10^{-8}$ & $4.86129 \times 10^{-8}$ & $4.6885 \times 10^{-8}$ & $3.63928 \times 10^{-8}$ \\
0.4 & $3.44902 \times 10^{-8}$ & $5.96351 \times 10^{-8}$ & $7.55814 \times 10^{-8}$ & $8.2482 \times 10^{-8}$ & $8.04953 \times 10^{-8}$ \\
0.5 & $4.31131 \times 10^{-8}$ & $7.65863 \times 10^{-8}$ & $1.00602 \times 10^{-7}$ & $1.15349 \times 10^{-7}$ & $1.21020 \times 10^{-7}$ \\
\hline
\end{tabular}

Table 4. $L_{2}$ and $L_{\infty}$ errors for Example (2) with $m=4,6$ and 10.

\begin{tabular}{ccccccc}
\hline$n$ & & \multicolumn{2}{c}{6} & \multicolumn{2}{c}{10} \\
\hline$x$ & $L_{2}$ & $L_{\infty}$ & $L_{2}$ & $L_{\infty}$ & $L_{2}$ & $L_{\infty}$ \\
\hline 0.1 & $4.34078 \times 10^{-6}$ & $1.3424 \times 10^{-6}$ & $1.37871 \times 10^{-6}$ & $4.32171 \times 10^{-7}$ & $4.61577 \times 10^{-7}$ & $1.48914 \times 10^{-7}$ \\
0.2 & $4.97100 \times 10^{-6}$ & $1.70414 \times 10^{-6}$ & $1.59318 \times 10^{-6}$ & $5.56933 \times 10^{-7}$ & $5.4413 \times 10^{-7}$ & $1.98079 \times 10^{-7}$ \\
0.3 & $5.60954 \times 10^{-6}$ & $2.0710 \times 10^{-6}$ & $1.80913 \times 10^{-6}$ & $6.83084 \times 10^{-7}$ & $6.26085 \times 10^{-7}$ & $2.47459 \times 10^{-7}$ \\
0.4 & $6.26191 \times 10^{-6}$ & $2.4466 \times 10^{-6}$ & $2.0284 \times 10^{-6}$ & $8.11842 \times 10^{-7}$ & $7.08138 \times 10^{7}$ & $2.97527 \times 10^{-7}$ \\
0.5 & $6.93387 \times 10^{-6}$ & $2.8344 \times 10^{-6}$ & $2.25293 \times 10^{-6}$ & $9.44397 \times 10^{-7}$ & $7.91008 \times 10^{-7}$ & $3.48733 \times 10^{-7}$ \\
\hline
\end{tabular}

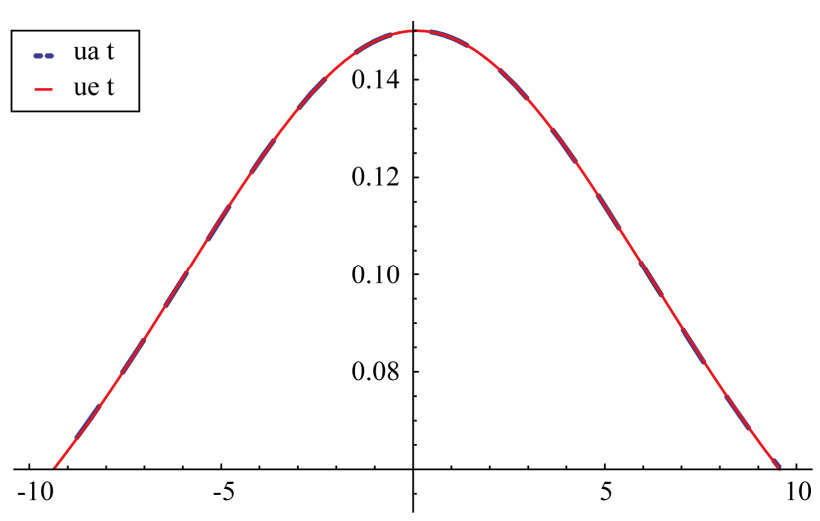

(a)

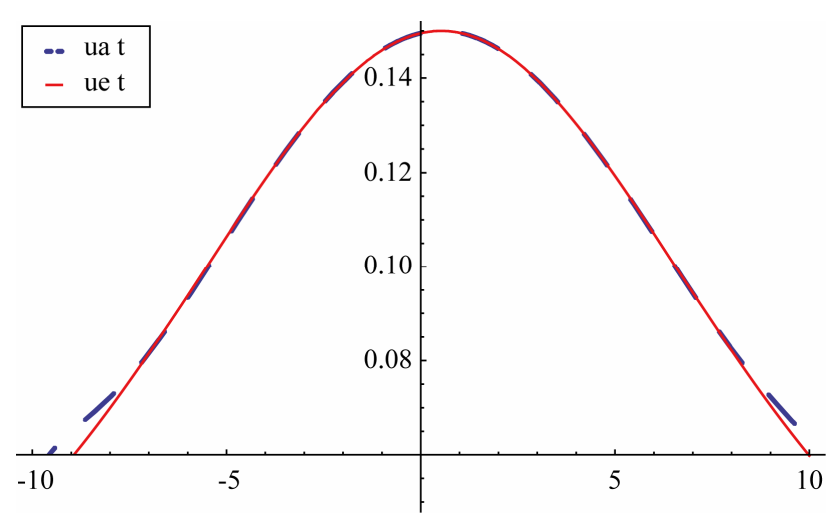

(b)

Figure 3. The exact solution and numerical solution with new modification of Laplace ADM for Example (1), (a) for $t=0.1$ and (b) for $t=0.5$. 


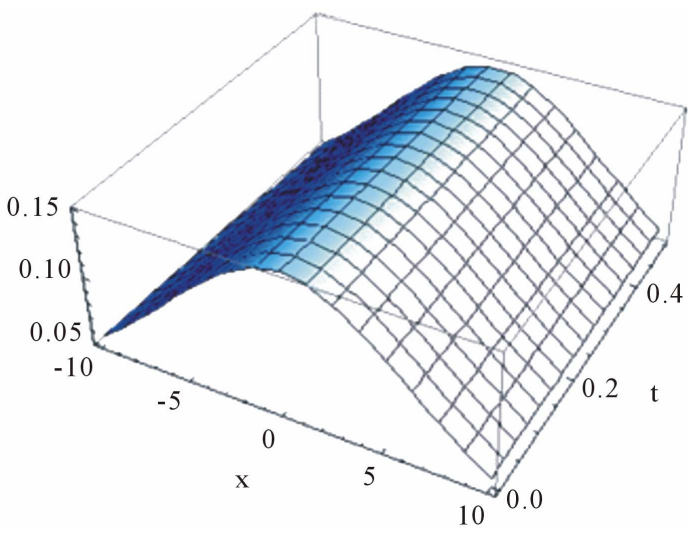

(a)

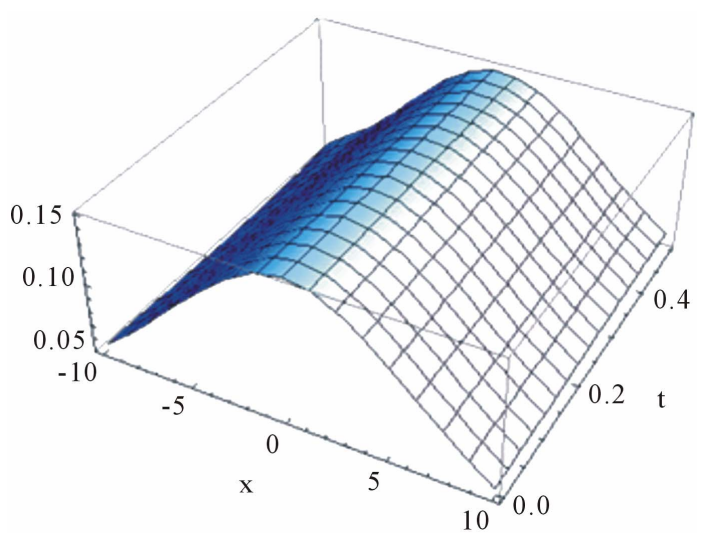

(b)

Figure 4. (a) The exact solution for $t=0.5$ and $-10 \leq x \leq 10$; (b) Numerical solution with new modification for $t=0.5$ and -10 $\leq x \leq 10$.

ADM to solve the RLW equation. The decomposition series solutions are converge very rapidly in real physical problems. The numerical results we obtained justify the advantage of this methodology, even in the few terms approximation is accurate. The method is tested on the problem of single solitary motion and high accuracy was achieved with the $L_{2}$ and $L_{\infty}$ error norms. The new Laplace ADM presented here is for the RLW equation, but it can be implemented to a large number of physically important nonlinear wave problems.

\section{REFERENCES}

[1] D. H. Peregrine, "Calculation of the Development of an Undular Bore," Journal of Fluid Mechanics, Vol. 25, No. 2, 1966, pp. 321-330. doi:10.1017/S0022112066001678

[2] Kh. O. Abdullove, H. Bogalubsky and V. G. Markhankov, "One More Example of Inelastic Soliton Interaction," Physics Letters A, Vol. 56, No. 6, 1976, pp. 427-428.

[3] J. L. Bona, W. G. Pritchard and L. R. Scott, "Numerical Schemes for a Model of Nonlinear Dispersive Waves," Journal of Computer Physics, Vol. 60, 1985, pp. 167-196.

[4] P. J. Jain and L. Iskandar, "Numerical Solutions of the Regularized Long Wave Equation," Computer Methods in Applied Mechanics and Engineering, Vol. 20, No. 2, 1979, pp. 195-201. doi:10.1016/0045-7825(79)90017-3

[5] L. R. T. Gardner, G. A. Gardner and A. Dogan, "A Least Squares Finite Element Scheme for RLW Equation," Communications in Numerical Methods in Engineering, Vol. 11, No. 1, 1995, pp. 59-68. doi:10.1002/cnm.1640110109

[6] İ. Dağ and M. N. Özer, "Approximation of the RLW Equation by the Least Square Cubic B-Spline Finite Ele- ment Method," Applied Mathematical Modelling, Vol. 25, No. 3, 2001, pp. 221-231. doi:10.1016/S0307-904X(00)00030-5

[7] Y. keskin and G. Oturanc, "Numerical Solution of Regularized Long Wave Equation by Reduced Differential Transform Method," Applied Mathematical Sciences, Vol. 4, No. 25, 2010, pp. 1221-1231.

[8] G. Adomian, "Nonlinear Stochastic Operator Equataions," Academic Press, San Diego, 1986.

[9] G. Adomian, "Solving Frontier Problem of Physics: The Decomposition Method," Kluwer Academic Publishers, Boston, 1994.

[10] E. Yusufoglu and A. Bekir, "Application of the Variational Iteration Method to the Regularized Long Wave Equation," Computer \& Mathematics with Applications, Vol. 25, 2003, pp. 321-330.

[11] G. Adomian, "Modified Adomian Polynomials," Mathematical Computation, Vol. 76, No. 1, 1996, pp. 95-97. doi:10.1016/0096-3003(95)00186-7

[12] A.-M. Wazwaz and S. M. El-Sayed, "A New Modification of the Adomian Decomposition Method for Linear and Nonlinear Operators," Applied Mathematics and Computation, Vol. 122, No. 3, 2001, pp. 393-405. doi:10.1016/S0096-3003(00)00060-6

[13] T. S. El-Danaf, M. A. Ramadan and F. E. I. AbdAlaal, "The Use of Adomian Decomposition Method for Solving the Regularized Long-Wave Equation," Chaos, Solitons \& Fractals, Vol. 26, No. 3, 2005, pp. 747-757. doi:10.1016/j.chaos.2005.02.012

[14] D. Kaya and S. M. El-Sayed, "An Application of the Decomposition Method for the Generalized KdV and RLW Equations," Chaos, Solitons \& Fractals, Vol. 17, No 5, 2003, pp. 869-877. doi:10.1016/S0960-0779(02)00569-6 